ARTICLE

Received 21 Mar 2014 | Accepted 9 Sep 2014 | Published 14 Nov $2014 \quad$ DOl: 10.1038/ncomms6210

\title{
Histone H3.3 and its proteolytically processed form drive a cellular senescence programme
}

Luis F. Duarte 1,2,3, Andrew R.J. Young ${ }^{4}$, Zichen Wang 3,5, Hsan-Au Wu1,3, Taniya Panda1,2, Yan Kou 3,5, Avnish Kapoor ${ }^{1,2, \dagger}$, Dan Hasson ${ }^{1,2}$, Nicholas R. Mills ${ }^{1,2}$, Avi Ma'ayan ${ }^{5}$, Masashi Narita ${ }^{4}$ \& Emily Bernstein ${ }^{1,2}$

The process of cellular senescence generates a repressive chromatin environment, however, the role of histone variants and histone proteolytic cleavage in senescence remains unclear. Here, using models of oncogene-induced and replicative senescence, we report novel histone $\mathrm{H} 3$ tail cleavage events mediated by the protease Cathepsin L. We find that cleaved forms of $\mathrm{H} 3$ are nucleosomal and the histone variant $\mathrm{H} 3.3$ is the preferred cleaved form of H3. Ectopic expression of $\mathrm{H} 3.3$ and its cleavage product ( $\mathrm{H} 3.3 \mathrm{cs} 1$ ), which lacks the first 21 amino acids of the H3 tail, is sufficient to induce senescence. Further, H3.3cs1 chromatin incorporation is mediated by the HUCA histone chaperone complex. Genome-wide transcriptional profiling revealed that $\mathrm{H} 3.3 \mathrm{cs} 1$ facilitates transcriptional silencing of cell cycle regulators including RB/E2F target genes, likely via the permanent removal of H3K4me3. Collectively, our study identifies histone $\mathrm{H} 3.3$ and its proteolytically processed forms as key regulators of cellular senescence.

\footnotetext{
${ }^{1}$ Department of Oncological Sciences, Icahn School of Medicine at Mount Sinai, One Gustave L. Levy Place, New York, New York 10029, USA. ${ }^{2}$ Department of Dermatology, Icahn School of Medicine at Mount Sinai, One Gustave L. Levy Place, New York, New York 10029, USA. ${ }^{3}$ Graduate School of Biomedical Sciences, Icahn School of Medicine at Mount Sinai, One Gustave L. Levy Place, New York, New York 10029, USA. ${ }^{4}$ Cancer Research UK Cambridge Institute, University of Cambridge, The Li Ka Shing Centre, Robinson Way, Cambridge CB2 ORE, UK. ${ }^{5}$ Department of Pharmacology and Systems Therapeutics and Systems Biology Center New York (SBCNY), Icahn School of Medicine at Mount Sinai, One Gustave L. Levy Place, New York, New York 10029, USA. $\dagger$ Present address: Department of Genomic Medicine, University of Texas MD Anderson Cancer Center, Houston, Texas 77030, USA. Correspondence and requests for materials should be addressed to E.B. (email emily.bernstein@mssm.edu).
} 
C ellular senescence is a stable form of growth arrest and is thought to function as a potent anti-tumour mechanism $^{1,2}$. Cellular senescence was originally defined as 'replicative exhaustion' that occurs in cultured cells over time, but can also be provoked prematurely by stresses that include DNA damage and activated oncogenes ${ }^{3-5}$. For example, melanocytes within human nevi often harbour an activating mutation in BRAF $\left(\mathrm{BRAF}^{\mathrm{V} 600 \mathrm{E}}\right)$ and can remain senescent for decades ${ }^{6}$. Characteristic features of senescent cells include morphological and physiological alterations, senescenceassociated $\beta$-galactosidase (SA- $\beta$-gal) activity, chromatin condensation and extensive gene expression changes ${ }^{4,7}$.

Senescence is mediated by the RB and p53 pathways, which cooperate to ensure cell cycle inhibition ${ }^{7-9}$. A growing body of evidence suggests that the process of cellular senescence is also mediated by chromatin changes ${ }^{10-15}$. Notably, senescent human cells often accumulate distinct 4',6-diamidino-2-phenylindole (DAPI)-dense nuclear foci known as senescent-associated heterochromatin foci (SAHF). SAHF are enriched for repressive chromatin modifications such as $\mathrm{H} 3 \mathrm{~K} 9 \mathrm{me} 3$ and $\mathrm{H} 3 \mathrm{~K} 27 \mathrm{me} 3$, chromatin architectural factors such as high mobility group A (HMGA) proteins, and the histone variant macroH $2 \mathrm{~A}^{11-13}$. Interestingly, SAHF formation is not driven by a redistribution of histone post-translational modifications (PTMs) in the senescent genome, but rather a spatial reorganization of existing repressive $\mathrm{PTMs}^{14}$. The deposition of macroH2A is a late step in SAHF formation and is dependent on components of the $\mathrm{H} 3.3$ histone chaperone complex HUCA (HIRA/UBN1/CABIN1/ASF1a) ${ }^{12}$. Interestingly, ectopic expression of HIRA or ASF1a alone or together can induce senescence; however, the role of H3.3 in driving a senescence programme remains unclear.

Recently, we mapped the histone PTM profile of senescent cells $^{15}$. We identified a striking loss of H3K4me3, a PTM associated with active transcription, at E2F target genes. We further identified the H3K4 demethylases JARID1A and JARID1B to be responsible for the removal of H3K4me3. In addition to the action of histone-modifying enzymes, the incorporation of histone variants and the proteolytic processing of histones have also emerged as mechanisms that alter the histone PTM landscape ${ }^{16}$. In fact, proteolytic processing of histone $\mathrm{H} 3$ has been reported in the heterochromatic micronuclei of Tetrahymena ${ }^{17}$ and during embryonic stem cell (ESC) differentiation via the lysosomal protease Cathepsin L1 $(\mathrm{CSTL} 1)^{18}$. However, a clear biological function for such histone tail processing has yet to be elucidated.

In this study, we report evidence of $\mathrm{H} 3$ tail proteolytic processing in models of both oncogene-induced and replicative senescence. We identified two distinct processing sites of the $\mathrm{H} 3$ tail, both of which are mediated by CTSL1. One of these sites, $\mathrm{H} 3 \mathrm{cs} 1$, corresponds to the cleavage site identified in differentiating ESCs, which generates a new $\mathrm{H} 3$ tail beginning at amino-acid T22 (ref. 18). We find a chromatin-bound pool of the active CTSL1 protease, which correlates with the emergence of cleaved $\mathrm{H} 3$ products during senescence. We demonstrate that the histone variant $\mathrm{H} 3.3$ is cleaved during senescence and that ectopic expression of $\mathrm{H} 3.3$, and to a greater extent $\mathrm{H} 3.3 \mathrm{cs} 1$, induces senescence in the absence of oncogenic signalling. Further, the chromatin incorporation of endogenous cleaved $\mathrm{H} 3$ during oncogene-induced senescence (OIS) and ectopic H3.3cs1 requires the HUCA complex. RNA-sequencing (RNA-seq) studies revealed that introduction of $\mathrm{H} 3.3 \mathrm{cs} 1$ into primary fibroblasts results in rapid transcriptional downregulation of cell cycle genes, with significant overlap of those downregulated during OIS. H3.3cs1-downregulated genes also significantly overlap with genes that lose $\mathrm{H} 3 \mathrm{~K} 4 \mathrm{me} 3$ during senescence, many of which are $\mathrm{RB}$ and JARID1A/1B targets ${ }^{15}$. Together, these findings suggest that $\mathrm{H} 3$ tail cleavage functions as a key mechanism to remove $\mathrm{H} 3 \mathrm{~K} 4 \mathrm{me} 3$, and thereby contributes to the silencing of cell cycle-promoting genes during senescence.

\section{Results}

Senescent fibroblasts show nucleosomal forms of cleaved $\mathbf{H 3}$. RAS-induced senescence (ER::H-RAS ${ }^{\mathrm{G} 12 \mathrm{~V}}$ ) in IMR90 fibroblasts is a well-characterized system in which senescence is enforced by the combined action of the RB and p53 tumour-suppressor pathways ${ }^{9,19}$. Using this system, we induced senescence by the addition of 4-hydroxytamoxifen (4OHT) to primary fibroblasts and assayed cells for established senescence markers (Fig. 1a; Supplementary Fig. 1). Consistent with our previous findings ${ }^{15}$, we detected a striking loss of $\mathrm{H} 3 \mathrm{~K} 4 \mathrm{me} 3$ during senescence (Fig. 1b). Upon immunoblotting for histone PTMs such as H3K14 and H3K18 acetylation, we noted not only an expected decrease of these marks, but also a faster running species of $\mathrm{H} 3$ (Fig. 1b, asterisks). Immunoblotting of PTMs more distal in the tail (H3K27me3 or H3K36me3) or with a C-terminal H3 antibody, revealed an additional faster-migrating species of $\mathrm{H} 3$ (Fig. 1b, arrows). These faster-migrating species appeared with increasing intensity over a senescence time course of $\sim 2$ weeks, but were not detected with antibodies specific for PTMs at the $\mathrm{N}$ terminus of the $\mathrm{H} 3$ tail, such as $\mathrm{H} 3 \mathrm{~K} 4 \mathrm{me} 3$ and $\mathrm{H} 3 \mathrm{~K} 9 \mathrm{me} 3$. No evidence of $\mathrm{H} 4$ cleavage was detected either (Fig. 1b). Collectively, these results suggested that the N-terminal tail of $\mathrm{H} 3$ is specifically cleaved during OIS at two distinct sites.

Using a combination of histone PTM-specific antibodies and an $\mathrm{H} 3$ cleavage-specific antibody $(\alpha \mathrm{H} 3 \mathrm{cs} 1$; ref. 18$)$, we aligned the putative cleavage sites of the $\mathrm{H} 3$ tail (Fig. 1c). Because the $\mathrm{H} 3 \mathrm{~K} 14 \mathrm{ac}$ antibody detects a faster migrating band that is not detectable with the $\mathrm{H} 3 \mathrm{~K} 9 \mathrm{me} 3$ antibody, the proximal cleavage site most likely lies at or within the K9-K14 stretch (see Fig. 1d). The distal cleavage site aligns with the $\mathrm{H} 3 \mathrm{cs} 1$ antibody, which was designed to recognize $\mathrm{H} 3$ with a $\mathrm{N}$ terminus beginning at $\mathrm{T} 22$ (Fig. 1c,d; H3csl epitope: TKAAR ${ }^{18}$ ). Therefore, we refer to the distal cleavage site as $\mathrm{H} 3 \mathrm{cs} 1$ and the proximal site as $\mathrm{H} 3 \mathrm{cs} 2$ (Fig. 1d). Owing to our ability to probe $\mathrm{H} 3 \mathrm{cs} 1$ with a specific antibody, we focused our studies on this distal cleavage site. Levels of $\mathrm{H} 3 \mathrm{cs} 1$ markedly increase upon OIS (Fig. 1e) and appear at much higher levels in senescent cells than mouse ESCs induced to differentiate (Supplementary Fig. 2).

We next investigated whether the cleaved $\mathrm{H} 3$ species were incorporated into chromatin and more specifically, nucleosomes. Growing and senescent IMR90s were fractionated into chromatin-free and chromatin fractions and probed with antibodies against $\mathrm{H} 3 \mathrm{cs} 1$ and the $\mathrm{C}$-terminal region of $\mathrm{H} 3$. Cleaved $\mathrm{H} 3$ products were detected only in the chromatin fraction of senescent cells (Fig. 1f). Next, soluble mononucleosomes were isolated from growing and senescent fibroblasts and immunoblotting revealed that cleaved $\mathrm{H} 3$ species are indeed nucleosomal in senescent cells (Fig. 1g). We highlight here that cleaved forms of the replication-independent histone variant $\mathrm{H} 3.3$ were also detected in these assays, suggesting that this histone variant may be preferentially processed in senescent cells (see below).

Senescent melanocytes exhibit cleaved forms of H3. We also investigated chromatin changes in human primary melanocytes induced to senesce. These specialized pigment cells can remain senescent in human nevi (moles) for decades, and have the potential to progress into melanoma. We induced senescence via serial passaging of melanocytes (replicative senescence) and using the activated oncogene $\mathrm{BRAF}^{\mathrm{V600E}}$ (OIS) ${ }^{6}$. Senescence was monitored via multiple established assays (Supplementary Fig. $3 \mathrm{a}-\mathrm{c})$. As evidenced in Fig. $1 \mathrm{~h}$, the histone $\mathrm{H} 3$ tail is 
a

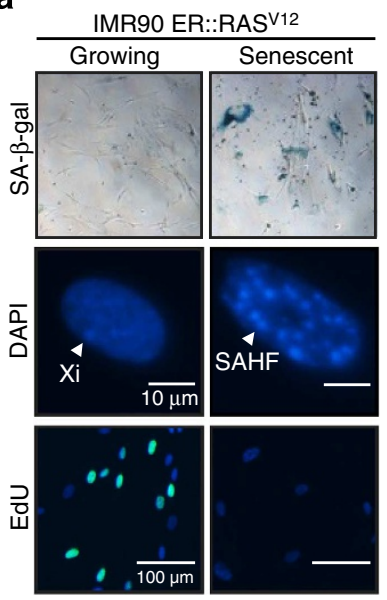

b

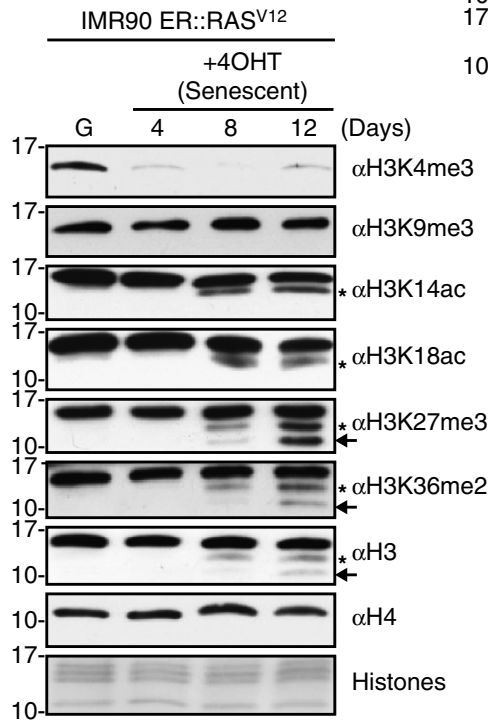

c

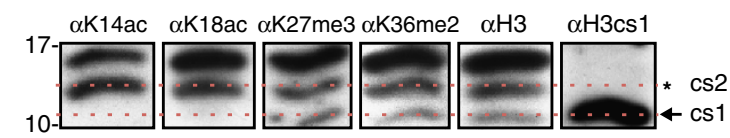

d

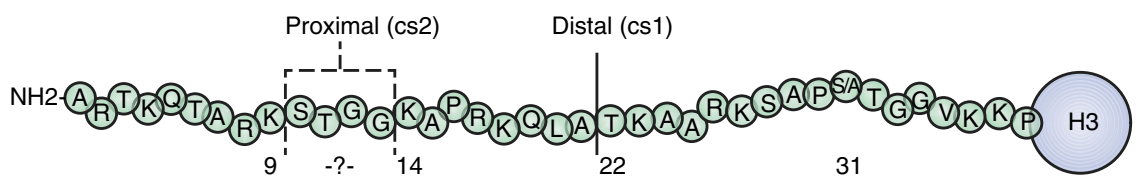

e

$\mathbf{f}$

g
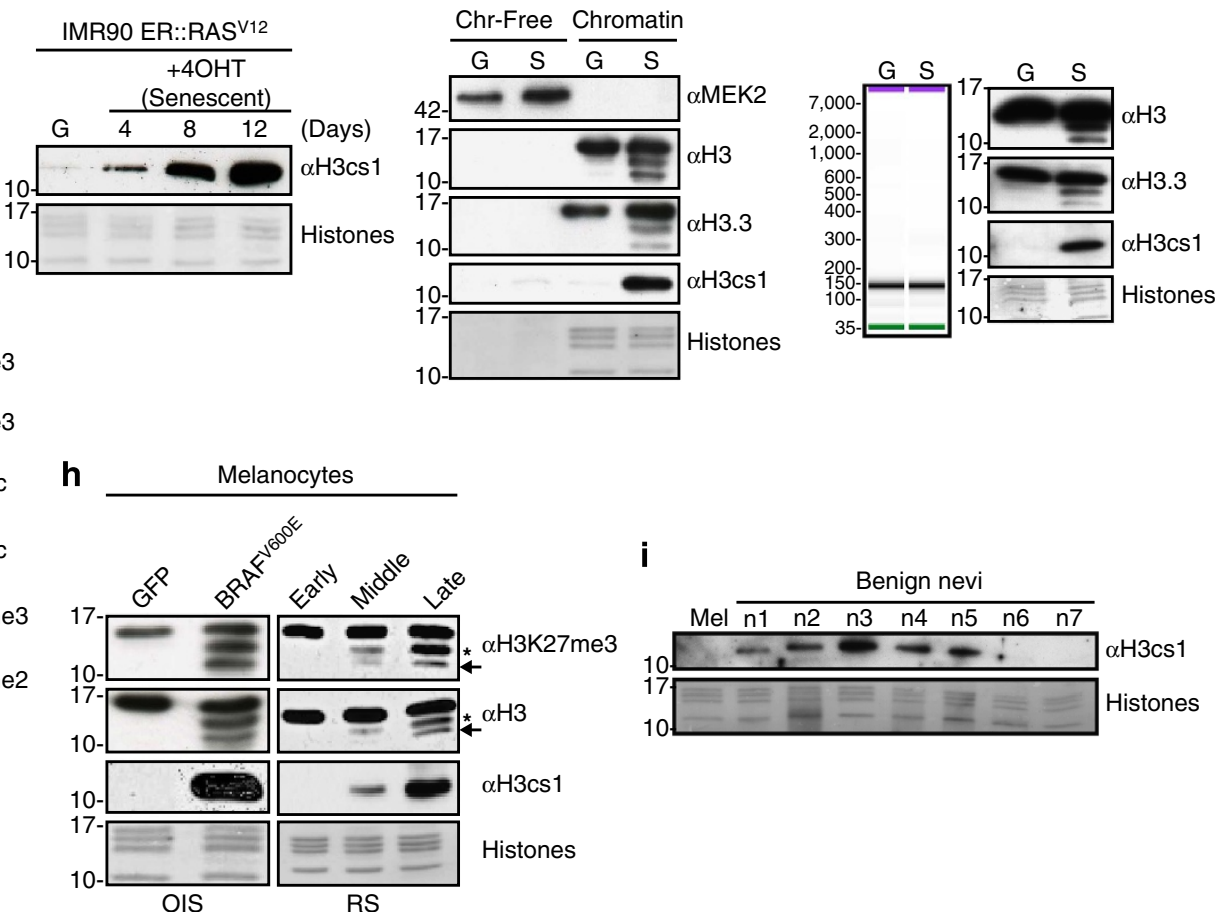

i

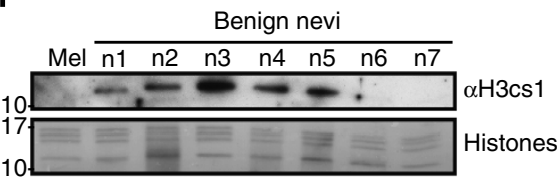

Figure 1 | Histone $\mathbf{H 3}$ tail is processed upon oncogene-induced senescence in fibroblasts and melanocytes. (a) Inducible RASV12-driven OIS system in IMR90 fibroblasts evidenced by SA- $\beta$-Gal staining ( $\times 10$ images shown), DAPI staining to monitor SAHF formation and EdU incorporation. Scale bars as shown. (b) Immunoblots of growing (G) and senescent (S) IMR90s with the indicated histone H3 PTM-specific antibodies. Asterisks indicate the proximal cleavage (cs2) and arrows indicate the distal cleavage (cs1). In all panels, core histones were stained with Amido Black for loading.

(c) Immunoblot of the chromatin fraction of senescent IMR90s showing alignment of full-length and cleaved histone H3 products. H3cs1-specific antibody used for alignment of the distal cleavage product. (d) Diagram of histone H3 tail showing cleavage sites. Dashed lines indicate potential proximal cleavage (cs2) sites, as determined by immunoblotting with H3 PTM-specific antibodies. The solid line marks the distal cleavage site (cs1). (e) Immunoblot of growing and senescent IMR9O cells with a cleavage-specific antibody ( $\alpha \mathrm{H} 3 \mathrm{cs} 1$ ), which recognizes the $\mathrm{N}$ terminus of H3 beginning at amino-acid T22 (ref. 18). (f) IMR90s (G and S) were fractionated into chromatin-free (cytoplasmic plus nuclear soluble) and chromatin fractions and blotted with the indicated antibodies. Mek2 used as a cytoplasmic control and histones/H3 used as chromatin controls. (g) (Left) bioanalyzer trace of chromatin from $\mathrm{G}$ and S IMR90s upon MNase digestion and DNA isolation shows mononucleosomes. Mononucleosomes were blotted with the indicated antibodies to confirm presence of cleaved histone $\mathrm{H} 3$ products. (h) Immunoblots of growing and senescent (oncogene-induced and replicative senescence) melanocytes with the indicated antibodies (early, middle and late represent passages 4, 6 and 8, respectively). Asterisks indicate the proximal cleavage (cs2) and arrows indicate the distal cleavage (cs1). (i) Immunoblot of acid-extracted histones from primary growing melanocytes (mel) and fresh frozen human benign nevi samples $(n=7)$ with $\alpha \mathrm{H} 3 \mathrm{cs} 1$.

cleaved during both modes of senescence in primary human melanocytes. Interestingly, the same pattern of processing observed in fibroblasts is detected in melanocytes, and the fastest migrating species corresponds to H3cs1 (Fig. 1h). Like fibroblasts, the cleaved forms of $\mathrm{H} 3$ in senescent melanocytes are chromatin associated (Supplementary Fig. 3d).

Next, we queried whether $\mathrm{H} 3 \mathrm{cs} 1$ could be a marker of senescence in vivo by probing acid-extracted histones isolated from fresh frozen benign nevi for $\mathrm{H} 3 \mathrm{cs} 1$. We identified the presence of $\mathrm{H} 3 \mathrm{cs} 1$ in the majority of benign nevi samples tested
(5/7), but not growing primary melanocytes (Fig. 1i). Further, by probing a panel of melanoma cell lines, we found that metastatic melanoma cells lack $\mathrm{H} 3 \mathrm{cs} 1$, while primary melanoma lines that fail to proliferate in culture contain $\mathrm{H} 3 \mathrm{cs} 1$ (Supplementary Fig. 3e). These findings suggest that $\mathrm{H} 3 \mathrm{cs} 1$ may be a useful marker for assessing senescence in premalignant lesions.

Processing of $\mathrm{H} 3$ requires activation of senescence programmes. We questioned whether oncogene activation or 
DNA damage, in the absence of senescence, could trigger $\mathrm{H} 3$ cleavage. First, we examined cells subjected to oncogenic stress but unable to undergo senescence. E1A-transduced ER::H-RAS ${ }^{\text {G12V }}$ IMR90s induced with 4OHT failed to enter senescence (Supplementary Fig. 4a,b), and were defective in $\mathrm{H} 3$ tail cleavage after 6 days of RAS ${ }^{\mathrm{G} 12 \mathrm{~V}}$ activation (Supplementary Fig. 4c). Moreover, acute induction of DNA damage via $24 \mathrm{~h}$ of etoposide treatment in IMR90s was not sufficient to induce $\mathrm{H} 3$ tail cleavage either (Supplementary Fig. 4d). Here, fibroblasts were arrested but not senescent as evidenced by various markers, including a lack of p16 expression (Supplementary Fig. 4d,e). These results strongly suggest that engagement of senescence effector pathways is required for the processing of $\mathrm{H} 3$.
CTSL1 cleaves $\mathrm{H3}$ and its inhibition impairs SAHF formation. The lysosomal protease CTSL1 localizes to the nucleus upon RAS activation ${ }^{20}$ and is responsible for cleaving the nuclear transcription factor $\mathrm{CDP} / \mathrm{Cux}$ and the $\mathrm{H} 3$ tail $^{18,21}$. To investigate whether CTSL1 is responsible for $\mathrm{H} 3$ tail cleavage during senescence, we first probed the levels of CTSL1 upon senescence induction in fibroblasts and melanocytes. CTSL1 levels increase transcriptionally and more strikingly at the protein level upon senescence in both systems (Fig. 2a-c). CTSL1 is found in three forms: the pro-cathepsin, intermediate and processed mature (active) forms ${ }^{22}$. Interestingly, our data show that the chromatin-bound pool of CTSL1 is enriched in the mature, active form of the protease, consistent with previous findings ${ }^{18}$. For example, at day 4 of senescence induction, only $\sim 17 \%$ of CTSL1 a

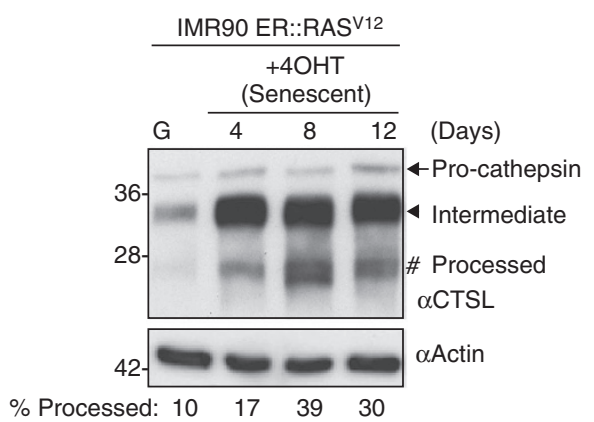

d

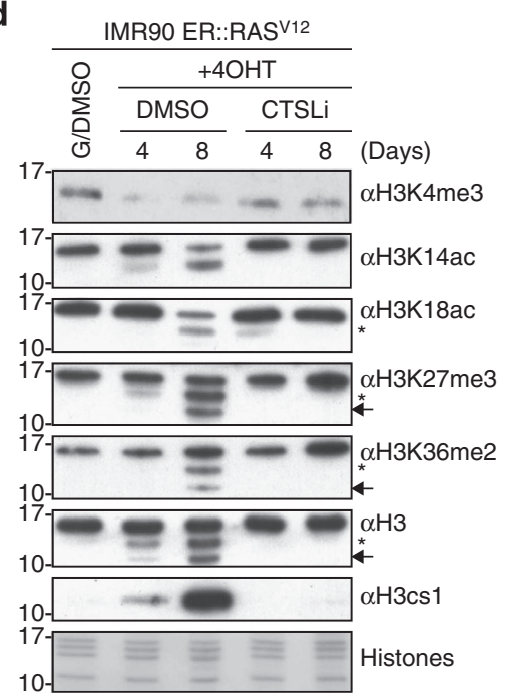

b
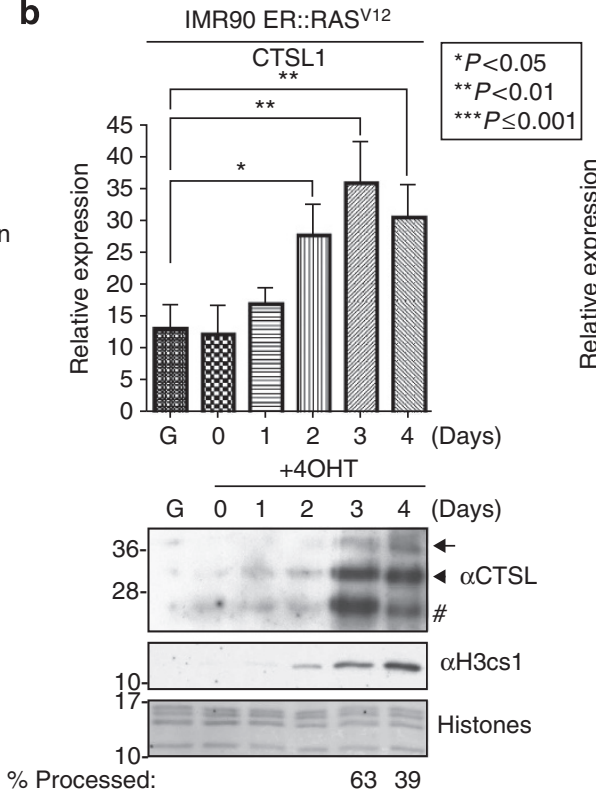

e

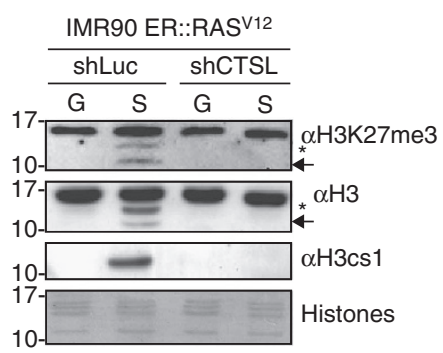

c
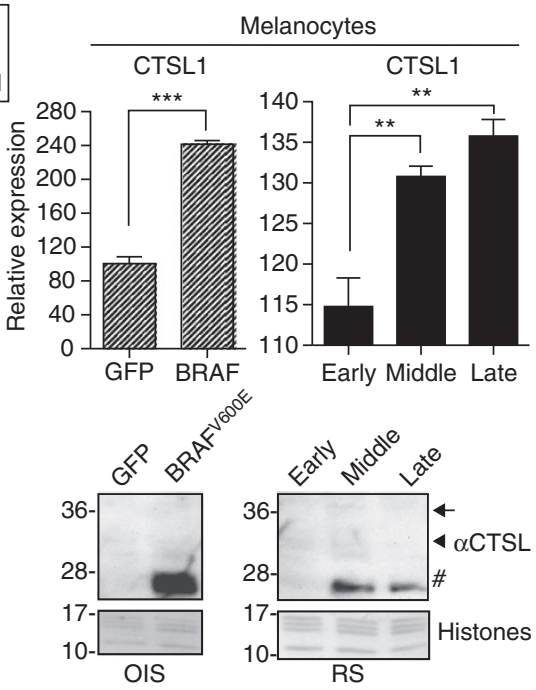

$\mathbf{f}$

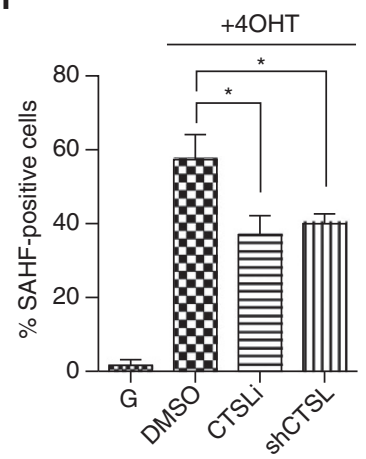

Figure 2 | Nuclear Cathepsin L mediates H3 tail processing and promotes SAHF. (a) Immunoblot for CTSL using whole-cell extract from growing and senescent IMR90s. Actin used for loading. (b) (Top) time course of CTSL1 induction as assessed by reverse transcription (RT)-quantitative (q)PCR. Values are normalized to expression of glyceraldehyde 3-phosphate dehydrogenase (GAPDH); mean \pm s.d. ( $n=3$ biological replicates); two-tailed unpaired Student's $t$-test; $P$ values as indicated. (Bottom) immunoblot of the chromatin fraction from growing and senescent IMR90 cells with the indicated antibodies. In all panels, core histones were stained with Amido Black for loading. Samples for RT-qPCR and immunoblot analysis were obtained at identical time points. Note correlation of CTSL1 transcriptional induction, presence of the active form of CTSL1 in the chromatin fraction and H3 tail cleavage. (c) (Top) relative expression of CTSL1 in growing and senescent melanocytes assessed by RT-qPCR. Values are normalized to expression of GAPDH; mean \pm s.d. ( $n=3$ technical replicates); two-tailed unpaired Student's $t$-test; $P$ values as indicated. (Bottom) immunoblot of CTSL using the chromatin fraction of growing and senescent melanocytes. (d) Chromatin extracts from growing and senescent IMR90 cells cultured in the presence of DMSO or a CTSL1 inhibitor probed with the indicated antibodies. Note the loss of H3 cleavage products in the CTSLi-treated cells, particularly at day 8. (e) Chromatin extracts from growing and senescent IMR90 cells expressing shLuc (control) or shCTSL1 lentiviral constructs probed with the indicated antibodies. Note the loss of $\mathrm{H} 3$ cleavage products in shCTSL1 senescent cells. (f) Quantification of SAHF in growing and senescent IMR90 cultured under the specified conditions obtained at day 8 post induction; mean \pm s.d. $\left(n=3\right.$ biological replicates); two-tailed unpaired Student's $t$-test; DMSO versus CTSLi ${ }^{\star} P=0.0147$; DMSO versus ShCTSL ${ }^{\star} P=0.0147$ 
exists in its mature form (Fig. 2a), while the chromatin-bound levels comprise $\sim 40 \%$ (Fig. 2b). In senescent melanocytes, we could only detect the mature form of CTSL1 in the chromatin fraction (Fig. 2c). Finally, a detailed time-course analysis of the early stages of senescence in IMR90s demonstrates that CTSL1 messenger RNA expression correlates with detection of active CTSL1 in the chromatin fraction, precisely when $\mathrm{H} 3$ tail cleavage is observed (Fig. 2b).

Next, we used both chemical inhibition (CTSLi) and short hairpin RNA (shRNA) approaches to assess the role of CTSL1 in processing the $\mathrm{H} 3$ tail. IMR90 ER::RAS ${ }^{\mathrm{V} 12}$ cells were induced to senesce with 4OHT in the presence of either dimethyl sulfoxide (DMSO) or a potent irreversible CTSL1-specific inhibitor (Fig. 2d). This inhibitor blocks self-processing of CTSL1 into its mature form, leading to the accumulation of the intermediate form (Supplementary Fig. 5a). In addition, ER::RAS ${ }^{\mathrm{V} 12}$-infected fibroblasts were engineered to express either shCTSL1 or shLuc control. This knockdown was specific for CTSL1 and was maintained over a 2-week course of senescence (Supplementary Fig. 5). In both scenarios, when cells were induced to senesce, we observed a block in the production of both $\mathrm{H} 3 \mathrm{cs} 1$ and $\mathrm{cs} 2$ (Fig. 2d,e). This implicates CTSL1 as the key protease responsible for the observed cleavage events. Of note, we observed that loss of $\mathrm{H} 3 \mathrm{~K} 4 \mathrm{me} 3$ is less pronounced upon CTSL inhibition as compared with DMSO-treated cells (Fig. 2d, and see below).

In addition, we queried whether CTSL1 inhibition, and thus a block in H3 cleavage, could bypass OIS. The inhibition of CTSL1 did not allow for increased proliferation in IMR90 ER:: RAS V12 cells induced to senesce (Supplementary Fig. 6), however, its inhibition/deficiency significantly impaired SAHF formation (Fig. 2f). While these data show that CTSL1 inhibition alone is not sufficient to bypass a robust senescence system such as RAS $^{\text {V12 }}$-driven OIS in IMR90s, they do suggest that CTSL1 activity and its downstream substrates (H3 and possibly others) are important for SAHF formation.

H3.3 and H3.3cs1 induce a senescence programme. Canonical $\mathrm{H} 3.1$ and the variant H3.3 differ by only five amino acids. Four of these residues cluster in the interface between $\mathrm{H} 3.3$ and $\mathrm{H} 4$, a region that mediates the interaction with distinct histone chaperone complexes ${ }^{23}$. The other differential amino acid is at position 31, which resides in the histone tail (Fig. 1d). To assess which $\mathrm{H} 3$ isoform is proteolytically processed during senescence, we first probed the chromatin fraction of growing and senescent cells with an antibody against $\mathrm{H} 3.3$ (which recognizes the $\mathrm{C}$ terminus of the histone). We verified this antibody is specific for H3.3 by immunoblotting tagged forms of $\mathrm{H} 3.1$ and $\mathrm{H} 3.3$ (Supplementary Fig. 7a). Using this antibody, we detected H3.3 cleavage products in senescent fibroblasts and melanocytes (Fig. 1f; Supplementary Fig. 4d). Moreover, H3.3 and its cleavage products are incorporated into nucleosomes (Fig. 1g). As previously reported ${ }^{10}$, we also find increased levels of $\mathrm{H} 3.3$ during a senescence time course (Supplementary Fig. 7b).

Next, we inquired whether ectopic expression of full-length forms of Flag-tagged H3.1 (H3.1FL) and H3.3 (H3.3FL) could induce senescence in IMR90s. As expected, these ectopically expressed histones are localized to the nucleus by immunofluorescence (Supplementary Fig. 7c). Intriguingly, by immunoblotting for Flag, we observed that H3.3 is cleaved with a similar pattern to that of OIS (Fig. 3a,b). Because of this finding, we next inquired whether expression of $\mathrm{H} 3.3 \mathrm{FL}$ or $\mathrm{H} 3.3 \mathrm{cs} 1$ was sufficient to induce senescence in IMR90s in the absence of oncogenic signalling. Thus, we generated H3.3cs1-Flag, which co-migrates with the faster migrating band of H3.3FL-Flag-cleavage products (Fig. 3b). Further supporting our investigation of H3.3cs1 in senescence, the identical construct generated for H3.1 (H3.1cs1) was not expressed or incorporated into chromatin (Supplementary Fig. $7 \mathrm{c}, \mathrm{d})$, suggesting that histone chaperone recognition was compromised or that this form of H3.1 may not be physiologically relevant.

Through a rigorous series of experiments, we found that H3.3cs1 induces senescence in fibroblasts compared with empty vector control (pBabe) and H3.1FL. Importantly, ectopic H3.3cs1 is expressed at similar levels to that of endogenous $\mathrm{H} 3 \mathrm{cs} 1$ during OIS (Supplementary Fig. 8a). H3.3FL can also induce senescence, albeit in a less robust manner than $\mathrm{H} 3.3 \mathrm{cs} 1$. Senescence induction in this system was monitored by cell morphology and SA- $\beta$-gal staining (Fig. 3c), incorporation of EdU to assess DNA replication (Fig. 3d), expression of CCNA (Cyclin A2), p16, p21 (Supplementary Fig. 8b-d) and SAHF formation (Fig. 3e). Consistent with a senescent phenotype, cells expressing H3.3FL and $\mathrm{H} 3.3 \mathrm{cs} 1$ showed larger nuclei than those expressing H3.1FL or pBabe (Fig. 3f, top panel). At higher magnification, DAPIdense SAHFs were clearly observed only in H3.3FL- and H3.3cs1expressing fibroblasts (Fig. 3f). Moreover, these SAHFs stained positively for macroH2A1, a late marker of SAHF formation ${ }^{12}$, suggestive of established SAHFs (Fig. 3f). Further, we find that $\mathrm{H} 3.3 \mathrm{FL}$ and $\mathrm{H} 3.3 \mathrm{cs} 1$ induce heterochromatization as evidenced by increased resistance to digestion with micrococcal nuclease (Fig. $3 \mathrm{~g}$ ) and changes in the PTM and histone variant profile consistent with senescence, including increased H4K20me3 and macroH2A levels (Fig. 3h).

Next we questioned whether H3.3L20A, a mutation of $\mathrm{H} 3$ that prevents CTSL1-mediated cleavage ${ }^{24}$, would induce senescence. Colony formation assays (Fig. 3i), quantitation of SA- $\beta$-gal staining and incorporation of EdU (Supplementary Fig. 9) showed that L20A induces a senescence phenotype similar to that of wild-type H3.3FL. These findings suggest that full-length H3.3 indeed plays a role in senescence independent of its proteolytic processing, which is not unexpected as senescent cells rely on DNA replicationindependent incorporation of histone H3.3 as they exit the cell cycle $^{25}$. Taken together, these results provide evidence that the predominant form of $\mathrm{H} 3$ proteolytically processed by CTSL1 during senescence is H3.3. Moreover, they demonstrate that H3.3 and more robustly its cleaved product ( $\mathrm{H} 3.3 \mathrm{cs} 1)$, but not $\mathrm{H} 3.1$, can drive a cellular senescence programme.

HUCA is required for chromatin incorporation of H3.3cs1. Next, we examined the localization of Flag-tagged H3.3FL and H3.3cs1 in IMR90s. H3.3FL and H3.3cs1 were found largely excluded from SAHF, which were marked by H3K9me3 (Fig. 4a). This is consistent with a recent study that also showed exclusion of $\mathrm{H} 3.3$ from $\mathrm{SAHF}^{25}$. These data further suggest that cleavage of $\mathrm{H} 3.3$ does not compromise $\mathrm{H} 3 \mathrm{~K} 9 \mathrm{me}$, which is likely to be found on canonical H3.1 or H3.2 in SAHF.

Because the ectopic expression of H3.3FL and H3.3cs1 demonstrated a largely euchromatic nuclear localization (Fig. 4a), we hypothesized that the HUCA histone chaperone complex may be responsible for H3.3cs1 deposition in senescent cells. The HUCA histone chaperone complex interacts specifically with H3.3, and is responsible for its deposition at distinct chromatin regions, including transcription start sites and the bodies of active genes ${ }^{26,27}$. To test whether this complex is required for chromatin incorporation of endogenous $\mathrm{H} 3 \mathrm{cs} 1$ during OIS senescence, IMR90 ER::RAS ${ }^{\mathrm{V} 12}$ cells were infected with lentiviral shRNAs against HIRA, ASF1a, UBN1 or luciferase control and induced to senesce. During the course of these studies, we noted that the HIRA knockdown was reproducibly incompatible with cell viability, and we therefore focused on knockdown of ASF1a and UBN1. Upon knockdown of either 
a

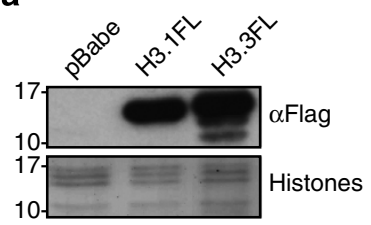

b

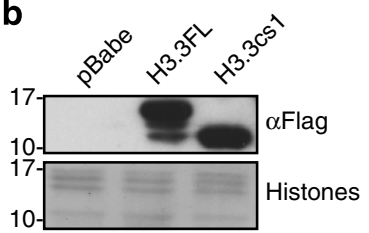

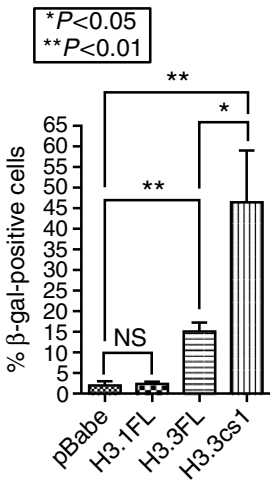

d

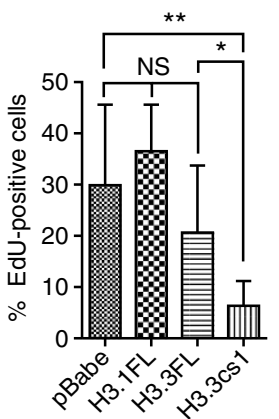

g

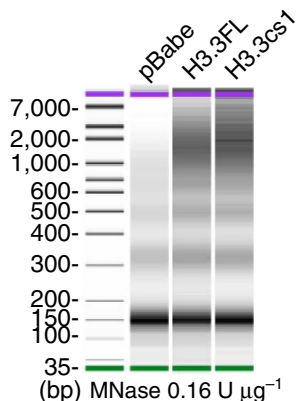

(bp) $\overline{\text { MNase } 0.16} \overline{\mathrm{U} \mu \mathrm{g}^{-1}}$

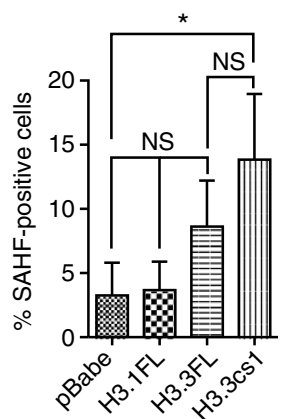

h
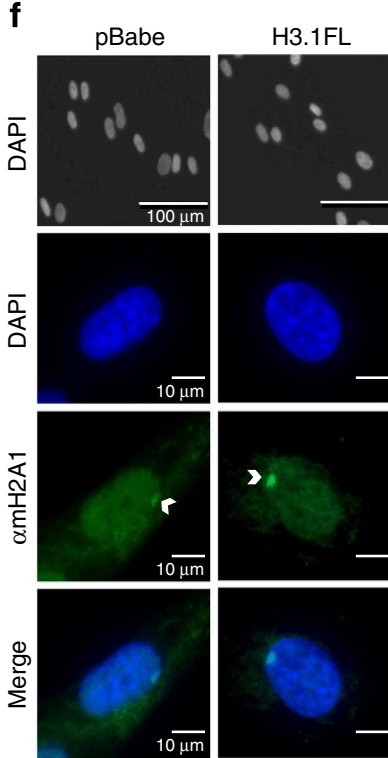

H3.3FL
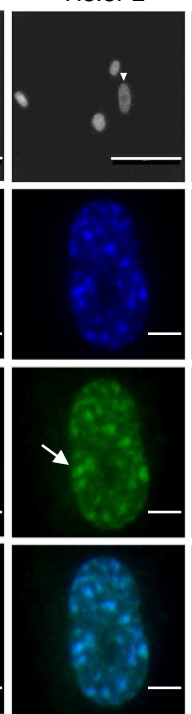

H3.3cs 1

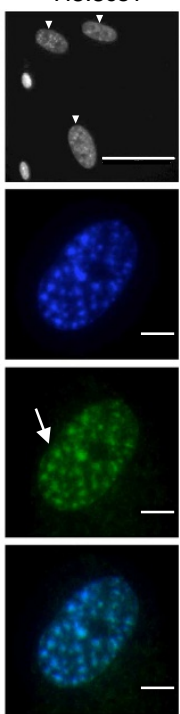

Figure 3 | Ectopic expression of H3.3 and H3.3cs1 induces senescence in fibroblasts. (a,b) Immunoblot of IMR90 cells stably expressing full-length H3.1 (H3.1FL), full-length H3.3 (H3.3FL), cleaved H3.3 (H3.3cs1) or empty pBabe vector with $\alpha$ Flag. Core histones stained with Amido Black for loading. Note the H3 cleavage products in H3.3FL-expressing cells. (c) Quantification of $\beta$-Gal-positive cells in IMR90 cells expressing the indicated histones; mean \pm s.d. ( $n=3$ biological replicates). (d) Quantification of EdU incorporation; mean \pm s.d. ( $n=3$ biological replicates). (e) Quantification of SAHF; mean \pm s.d. ( $n=3$ biological replicates); $P$ values for c-e are as indicated; two-tailed unpaired Student's t-test. (f) (Top) DAPI-stained nuclei of IMR90s expressing the indicated histones. Scale bars as shown. (Middle) immunofluorescence of IMR90s expressing the indicated histones stained with DAPI and $\alpha$ macroH2A1. Arrowheads indicate Xi and arrows indicate SAHF. (Bottom). Merged images of DAPI and $\alpha$ macroH2A1. Images taken at $\times 40$ magnification. (g) Nuclei obtained from IMR90s expressing the indicated histones were digested with MNase at 0.16 units per $\mu$ g of chromatin. DNA was isolated after MNase digestion and analysed by a high-sensitivity DNA assay (Agilent 2100 Bioanalyzer). (h) Chromatin fraction from IMR90 cells expressing pBabe, H3.3FL and H3.3cs1 probed with the indicated antibodies. Core histones stained with Amido Black for loading. (i) Colony formation assay course (day 16 post infection) of IMR90s expressing the indicated histones. NS, not significant.

ASF1a or UBN1 (Fig. 4b; Supplementary Fig. 10a), we observed a marked reduction in the incorporation of $\mathrm{H} 3 \mathrm{cs} 1$ in the chromatin fraction compared with control cells (Fig. 4c). This was specific for HUCA, as knockdown of Daxx, a component of a separate chaperone that deposits $\mathrm{H} 3.3$ at telomeres ${ }^{28,29}$, did not affect H3cs1 levels in chromatin (Supplementary Fig. 10b,c).

To complement these findings, we investigated whether ASF1a mediates the ability of ectopically expressed $\mathrm{H} 3.3 \mathrm{cs} 1$ to induce senescence in IMR90s in the absence of oncogenic signalling. We generated an $\mathrm{H} 3.3 \mathrm{cs} 1$-Flag construct with an R-to-E substitution at position 129 (R129E), a mutation that has been shown to disrupt interaction of H3.3 with ASF1a (ref. 23). We also generated $\mathrm{H} 3.3 \mathrm{cs} 1$-Flag containing an $\mathrm{S}$-to-A substitution at position 31 (S31A), which mimics the tail of $\mathrm{H} 3.1$ and is not likely to alter chaperone binding. IMR90 cells were transduced with pBabe, H3.3cs1, H3.3cs1 S31A and H3.3cs1 R129E (Fig. 4d).
Immunoblots with $\alpha$-Flag demonstrate that while H3.3cs 1 and $\mathrm{H} 3.3 \mathrm{cs} 1 \mathrm{~S} 31 \mathrm{~A}$ are incorporated into chromatin, H3.3cs1 R129E is not (Fig. 4d). Consistent with these findings, H3.3cs1 S31A induces senescence as efficiently as $\mathrm{H} 3.3 \mathrm{cs} 1$, as measured by changes in cell morphology and increased SA- $\beta$-gal staining (Fig. 4e), loss of EdU incorporation (Fig. 4f), induction of SAHF formation (Fig. 4g), decreased CCNA expression (Fig. 4h) and induction of p16 (Fig. 4i). On the other hand, H3.3cs1 R129E failed to induce senescence markers in IMR90 cells by all accounts (Fig. 4e-i). Collectively, these data suggest that ASF1a and $\mathrm{UBN} 1$, via direct interaction with $\mathrm{H} 3.3$, are required for incorporation of cleaved H3.3 into chromatin.

$\mathrm{H} 3.3 \mathrm{cs} 1$ represses E2F target genes via $\mathrm{H} 3 \mathrm{~K} 4 \mathrm{me} 3$ loss. To gain a better understanding of the gene expression programme mediated 
a

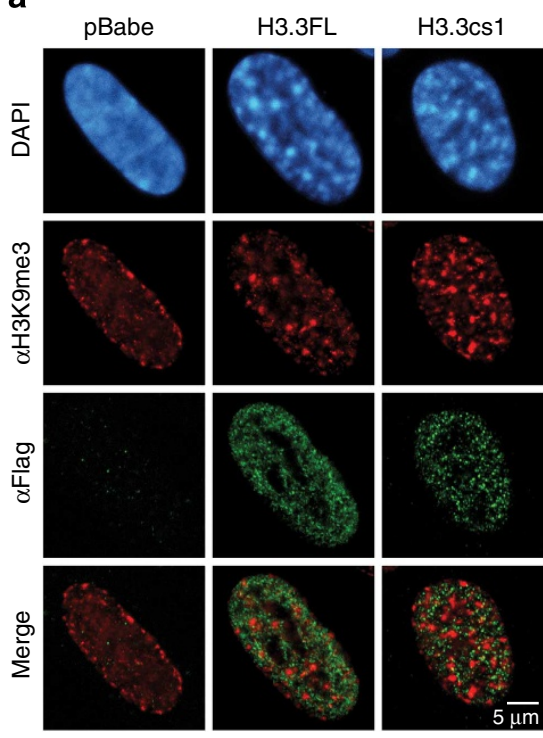

e b

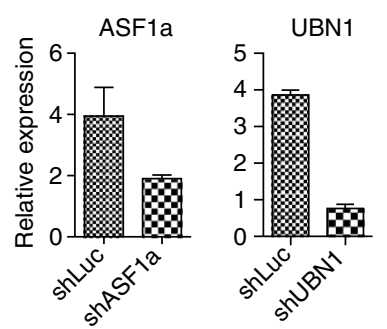

C

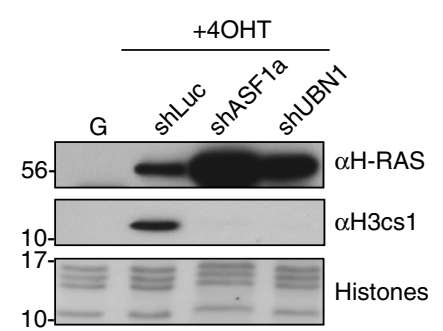

d

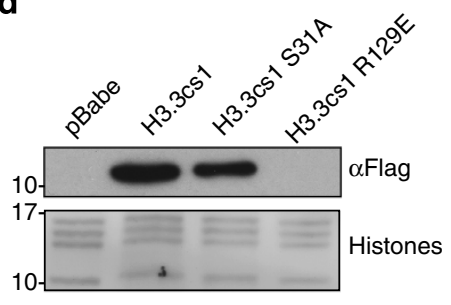

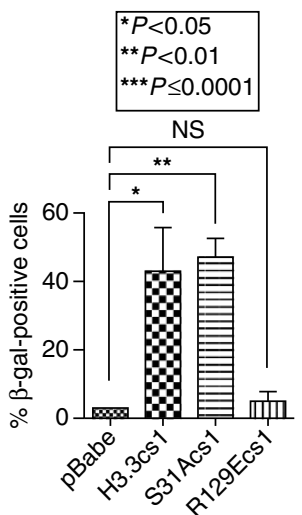

f

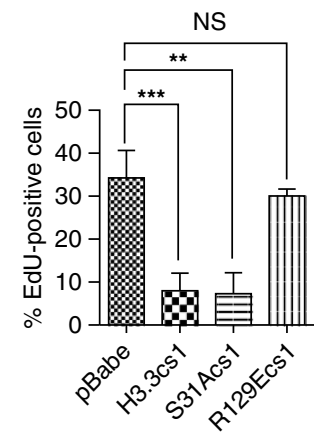

g

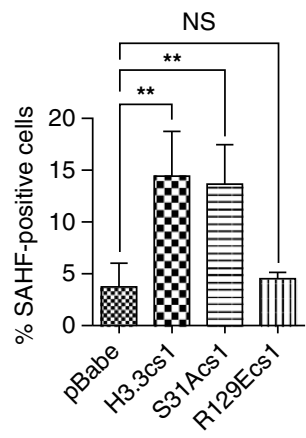

h

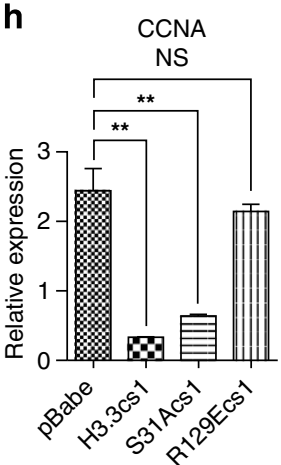

i p16

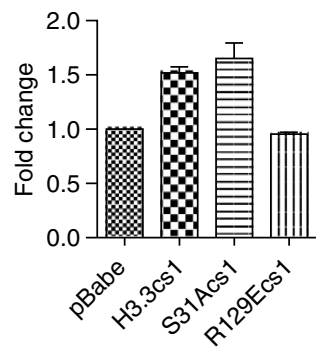

Figure 4 | The histone chaperone complex HUCA is required for chromatin incorporation of H3.3cs1. (a) Confocal microscopy of Flag-tagged H3.3FL and H3.3cs1. DAPI, H3K9me3 (red), Flag (green) staining and merged (red and green) images shown. (b) Confirmation of ASF1a and UBN1 knockdown in IMR90 ER::RASV12 cells by reverse transcription-quantitative PCR. Values are normalized to expression of glyceraldehyde 3-phosphate dehydrogenase (GAPDH); mean \pm s.d. ( $n=2$ biological replicates for ASF1a and $n=3$ technical replicates for UBN1). (c) Chromatin fraction from growing and senescent IMR90 ER::RASV12 cells expressing either shLuc, shASF1a or shUBN1 probed with $\alpha \mathrm{H} 3 \mathrm{cs} 1 . \alpha \mathrm{H}$-RAS immunoblot shows inducible expression of RASV12. Core histones stained with Amido Black for loading. (d) Immunoblot of the chromatin fraction of IMR90 cells expressing the indicated histones with $\alpha$ Flag. (e) Representative SA- $\beta$-Gal staining images of IMR90 expressing the indicated histones ( $\times 10$ images shown); quantification shown on the right; mean \pm s.d. ( $n=2$ biological replicates with three technical replicates). ( $\mathbf{f}-\mathbf{i})$ Quantification of EdU incorporation, SAHF-positive cells, CCNA and p16 levels in IMR90 cells expressing the indicated histones. Values are normalized to expression of GAPDH; mean \pm s.d. ( $n=3$ technical replicates); $P$ values for $\mathbf{e}-\mathbf{i}$ as indicated; two-tailed unpaired Student's $t$-test. For p16, expression is relative to pBabe control. NS, not significant.

by cleaved H3.3, we performed RNA-seq of growing and OIS fibroblasts, as well as fibroblasts expressing H3.3cs1 at two different time points post infection (early: day 3 and late: day 13). As expected, we found a marked change in the gene expression programme of OIS as compared with growing cells (Fig. 5a; Supplementary Table 1). Interestingly, we noted that the majority of the differentially expressed genes in the H3.3cs1-induced senescence samples at both early and late time points were downregulated when compared with growing cells. We found a striking significant overlap between these downregulated genes and genes downregulated in OIS (Fig. 5a,b), suggesting that similar pathways are repressed in both systems. Consistent with our previous findings ${ }^{15}$, Gene Ontology (GO) analysis reveals that OIS and H3.3cs1-downregulated genes are similarly involved in cell cycle progression (Supplementary Fig. 11a). To understand transcription factor enrichment for the differentially expressed downregulated genes during both OIS and H3.3cs1-induced senescence, we used the ChIP-X Enrichment Analysis tool (ChEA), which contains gene lists for 205 different mammalian transcription factors extracted from over 230 chromatin 
a
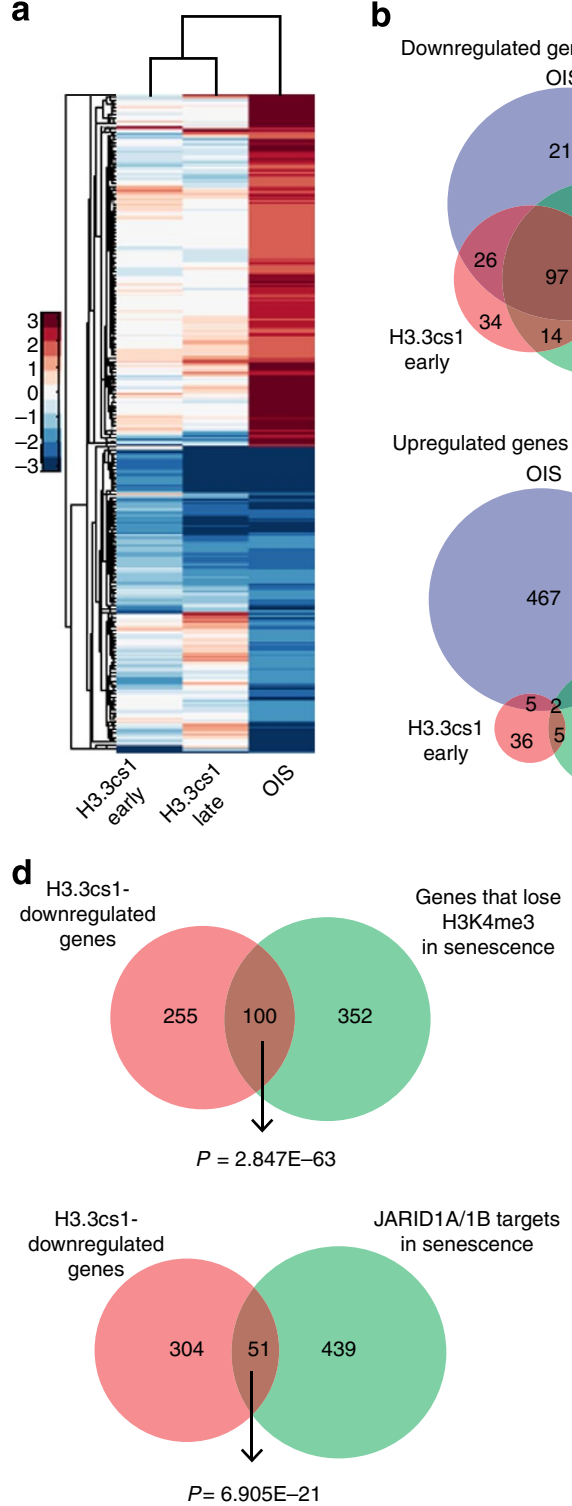

C

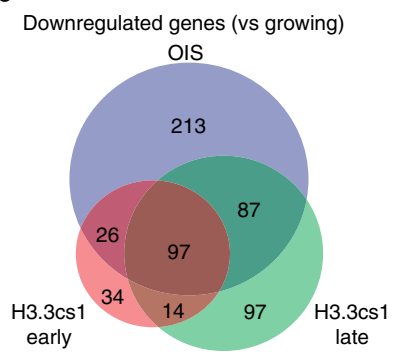

Upregulated genes (vs growing)

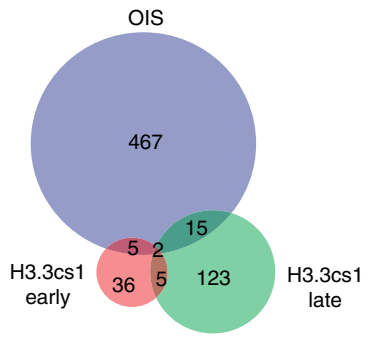

ChEA : H3.3cs1 downregulated genes - Log ( $P$-value) $\begin{array}{llllllllllll}0 & 10 & 20 & 30 & 40 & 50 & 60 & 70 & 80 & 90 & 100\end{array}$

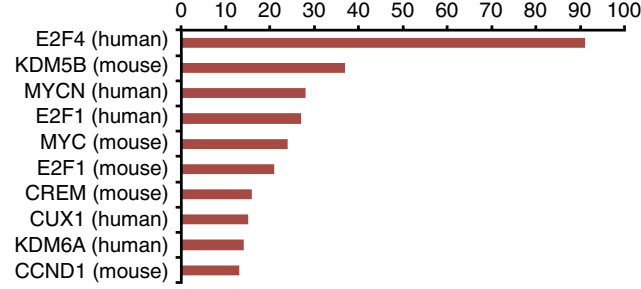

ChEA : OIS downregulated genes -Log ( $P$-value $)$

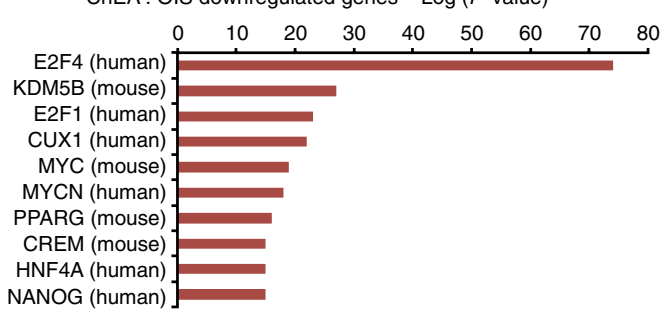

e

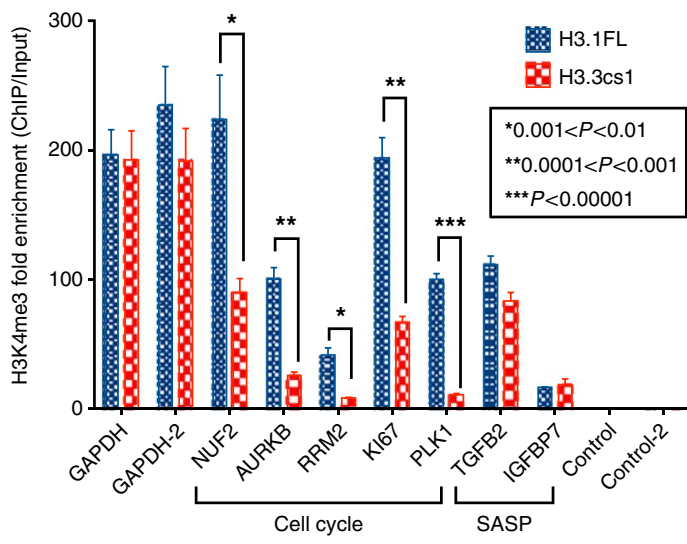

Figure 5 | H3.3cs1-induced senescence is mediated by decreased transcriptional output of cell cycle genes and reduced H3K4me3. (a) Heat map showing gene expression changes of 914 genes as determined by RNA-seq in H3.3cs1-induced senescence (early and late time points) relative to those that are altered in OIS (versus growing). Note the overlap of downregulated genes between H3.3cs1-induced senescence and OIS. (b) (Top) venn diagram of genes downregulated in OIS, H3.3cs1 (early) and H3.3cs1 (late) as compared with growing IMR90s. Fisher exact test; $P=2.418 \mathrm{E}-117$ OIS versus $\mathrm{H} 3.3 \mathrm{cs} 1$ (early); $P=3.477 \mathrm{E}-162$ OIS versus H3.3cs1 (late); $P=2.404 \mathrm{E}-117 \mathrm{H} 3.3 \mathrm{cs} 1$ (early) versus H3.3cs1 (late). (Bottom) venn diagram of genes upregulated in OIS, H3.3cs1 (early) and H3.3cs1 (late) as compared with growing IMR90s. Fisher exact test; $P=3.414 \mathrm{E}-4$ OIS versus H3.3cs1 (early); $P=2.883 \mathrm{E}-7$ OIS versus $\mathrm{H} 3.3 \mathrm{cs} 1$ (late); $P=1.783 \mathrm{E}-7 \mathrm{H} 3.3 \mathrm{cs} 1$ (early) versus H3.3cs1 (late). (c) ChIP Enrichment Analysis (ChEA) of H3.3cs1-induced senescence (top) and OIS (bottom)-downregulated genes. Note that E2F family members and KDM5B (JARID1B) gene targets are enriched in both. The $-\log$ of the $P$ value associated with each term is plotted. (d) Venn diagrams illustrating the overlap between H3.3cs1-downregulated genes and genes that lose H3K4me3 in senescence and JARID1A/1B-regulated genes in senescence ${ }^{15}$. Fisher exact test of $P$ values indicated in the diagram.

(e) ChIP-quantitative PCR for H3K4me3 at cell cycle genes and SASP-related genes in IMR90s expressing H3.1FL (blue) or H3.3cs1 (red). Two regions near the glyceraldehyde 3-phosphate dehydrogenase (GAPDH) promoter used as positive controls; intergenic regions used as negative controls. Fold enrichment of H3K4me3 ChIP/input is plotted. Mean \pm s.e.m.; two-tailed paired sample $t$-test; $P$ values as indicated.

immunoprecipitation-sequencing (ChIP-seq) or ChIP-chip publications ${ }^{30}$. This analysis confirms that these downregulated genes are targets of a common set of transcription factors and epigenetic regulators, including members of the E2F family and the H3K4me3 demethylase KDM5B (JARID1B) (Fig. 5c).

Previously, we reported that RB and JARID1A/1B cooperate in the silencing of E2F targets during senescence via H3K4me3 demethylation $^{15}$. To investigate a potential role for $\mathrm{H} 3$ tail cleavage in the removal of $\mathrm{H} 3 \mathrm{~K} 4 \mathrm{me} 3$, we compared our RNA-seq results with the ChIP-seq and gene expression data from that study. We found a significant overlap between $\mathrm{H} 3.3 \mathrm{cs} 1-$ downregulated genes and those genes in which $\mathrm{H} 3 \mathrm{~K} 4 \mathrm{me} 3$ is lost during senescence (Fig. 5d, top). Furthermore, the overlap between H3.3cs1-downregulated genes and those regulated by JARID1A/1B (Fig. 5d, bottom) and RB (Supplementary Fig. 11b) is highly significant.

Next, we performed ChIP for H3K4me3 in IMR90s expressing either H3.1FL (which does not induce senescence) or H3.3cs1 


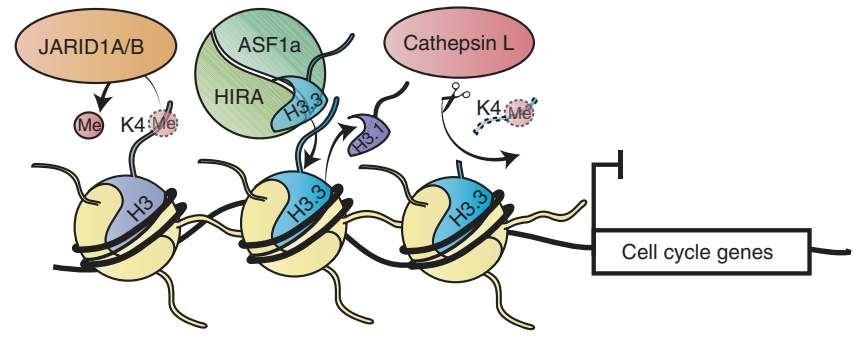

Figure 6 | Model depicting the mechanisms of H3K4me3 loss in senescence. The HUCA histone chaperone complex showing ASF1a/HIRA is depicted depositing H3.3, coupled to eviction of replication-dependent histone H3.1. Both JARID1A/1B-mediated demethylation and CTSLmediated proteolytic processing of $\mathrm{H} 3.3$ tail act to prevent transcription of cell cycle-related genes via removal of $\mathrm{H} 3 \mathrm{~K} 4 \mathrm{me} 3$. These processes may work together or independently to ensure the silencing of proliferationpromoting genes during cellular senescence.

(which induces senescence). We focused on the promoters of representative cell cycle-promoting genes, senescence-associated secretory phenotype (SASP)-related genes (see below), glyceraldehyde 3-phosphate dehydrogenase (not transcriptionally altered upon senescence), as well as intergenic regions that are not occupied by $\mathrm{H} 3 \mathrm{~K} 4 \mathrm{me} 3$ as negative controls (Fig. 5e). We found that cell cycle genes that are transcriptionally downregulated during $\mathrm{H} 3.3 \mathrm{cs} 1$-induced senescence (via RNA-seq) show significantly less $\mathrm{H} 3 \mathrm{~K} 4 \mathrm{me} 3$ at their promoters as compared with H3.1FL-expressing cells. Of note, these representative genes are also downregulated in OIS, but are not reported targets of the JARID1A/1B demethylases ${ }^{15}$. Together, these data support a model in which H3.3 tail cleavage functions in a similar manner as the JARID1 demethylases to remove $\mathrm{H} 3 \mathrm{~K} 4 \mathrm{me} 3$ and facilitate silencing of proliferation-promoting $\mathrm{RB} / \mathrm{E} 2 \mathrm{~F}$ target genes during senescence (Fig. 6). It further suggests that the proteolytic processing of the $\mathrm{H} 3.3$ tail functions as a key mechanism for $\mathrm{H} 3 \mathrm{~K} 4 \mathrm{me} 3$ loss at a subset of genes that are downregulated during senescence. This is supported by the fact that CTSL inhibition partially impedes the loss of $\mathrm{H} 3 \mathrm{~K} 4 \mathrm{me} 3$ in senescent cells (Fig. 2d, top blot).

Finally, we observed that a much larger set of genes is upregulated during OIS than during $\mathrm{H} 3.3 \mathrm{cs} 1$-induced senescence (early and late) compared with growing cells (Fig. 5a), and that there is little overlap between these groups (Fig. 5b, bottom). GO analysis of the upregulated genes in each system reveals that in fact different biological processes are affected (Supplementary Fig. 11c,d). Fibroblasts undergoing OIS display induction of cell signalling, inflammatory and secretory pathways that play a major role in the $\mathrm{SASP}^{7,31,32}$. This includes interleukin 6, interleukin 8, matrix metalloproteinase 3 and CXCL1, among others (see Supplementary Table 1). Interestingly, H3.3cs1-induced senescence appears largely deficient in SASP induction, which may explain in part the milder senescence phenotype observed as compared with $\mathrm{RAS}^{\mathrm{V} 12}$-induced senescence. We note, however, that some SASP-like factors such as IGFBP2, 3, 7 and transforming growth factor $\beta 2$ were induced upon $\mathrm{H} 3.3 \mathrm{cs} 1$ ectopic expression. While heterogeneous in nature, multiple lines of evidence suggest that a persistent DNA damage response is required to trigger $\mathrm{SASP}^{32,33}$. Consistent with this, we found that while OIS triggers a DNA-damage response as previously reported ${ }^{34,35}$, H3.3cs1 overexpression fails to do so (Supplementary Fig. 12).

\section{Discussion}

Although senescence is associated with marked reorganization of chromatin structure, the distinct mechanistic chromatin-mediated events that contribute to this process remain poorly understood. Here we uncover novel senescence-associated proteolytic processing of histone $\mathrm{H} 3$ mediated by CTSL1 in human fibroblasts and melanocytes. CTSL1 cleaves the histone $\mathrm{H} 3$ tail at least at two distinct sites generating new $\mathrm{N}$ termini at residues $\mathrm{T} 22(\mathrm{H} 3 \mathrm{cs} 1)$, as previously reported in differentiating mouse $\mathrm{ESCs}^{18}$, and a novel site likely between $\mathrm{K} 9$ and $\mathrm{K} 14$ ( $\mathrm{H} 3 \mathrm{cs} 2)$. Of note, $\mathrm{H} 3 \mathrm{cs} 2$ was not identified in ESCs upon differentiation ${ }^{18}$, and thus appears specific for cellular senescence. It will be of interest in the future to map the precise $\mathrm{N}$ terminus of $\mathrm{H} 3 \mathrm{cs} 2$, determine whether the $\mathrm{cs} 1$ and cs2 cleavage events occur sequentially or simultaneously and to investigate whether $\mathrm{H} 3 \mathrm{cs} 2$ can also induce senescence.

Recently, a study showed evidence of senescence-associated H3 tail cleavage, however, this was reported to be a cytoplasmic event $^{36}$. In that study, H3 tail processing was due to chromatin fragments budding out of the nucleus as a consequence of disrupted nuclear envelope integrity. In turn, histones could be processed by lysosomes and progressively depleted. We have no evidence to suggest cytoplasmic processing of $\mathrm{H} 3$ in our systems and demonstrate that cleaved forms of $\mathrm{H} 3$ are indeed associated with chromatin and incorporated into nucleosomes. Moreover, the presence of active CTSL1 in the chromatin fraction of senescent cells is consistent with findings from other systems ${ }^{18,20,21,37}$. However, it remains plausible that a small fraction of cytoplasmic $\mathrm{H} 3 \mathrm{cs} 1$ was not detectable by our biochemical methods or that the appearance of such cytoplasmic histone processing occurs at later time points of senescence than we have assayed.

Because H3.3 is deposited into chromatin in a DNA replication-independent manner, it is likely that non-dividing cells, such as those undergoing senescence, depend on incorporation of H3.3. In fact, a recent study shows that while newly synthesized H3.3 is actively deposited into chromatin of senescent cells, H3.1 is not ${ }^{25}$. Consistent with this idea, we and others have found increased levels of H3.3 in the chromatin template of senescent cells (Supplementary Fig. 7b; reviewed in ref. 10). We also found that the ectopic expression of $\mathrm{H} 3.3$, but not H3.1, induces a senescence programme in fibroblasts characterized by classic markers of bona fide senescence, including increased SA- $\beta$-Gal activity and formation of macroH2A-containing SAHF (Fig. 3). The observed senescence phenotype is more robust with cleaved H3.3 ( $\mathrm{H} 3.3 \mathrm{cs} 1)$ than the full-length histone, suggesting that the proteolytic processing of H3.3 is particularly important.

The fact that the ASF1a and UBN1 are required for the chromatin incorporation of $\mathrm{H} 3 \mathrm{cs} 1$ during senescence is consistent with studies reporting a role for HIRA and ASF1a in senescence $^{12}$. We further demonstrate that the ability of ectopic $\mathrm{H} 3.3 \mathrm{cs} 1$ to induce senescence is dependent on its ability to directly interact with ASF1a (Fig. 4). Because HIRA knockdown is incompatible with fibroblast viability, we cannot exclude a role for HIRA in H3.3cs1 deposition, and suggest that it may play distinct roles in proliferating versus senescent cells. Interestingly, while histone chaperone complexes have preferred histone targets (for example, HUCA with H3.3 and HJURP with CENPA), evidence to date suggests that upon altered cellular conditions, alternate chaperone complexes can deposit histones if the preferred factor is not available or the histone is overexpressed ${ }^{27,28,38}$. However, our data suggest that HUCA is unique in its ability to incorporate $\mathrm{H} 3.3 \mathrm{cs} 1$ into chromatin, as other chaperones could not compensate in its absence or for a disruption of the H3.3-ASF1a interaction in IMR90 cells.

While HUCA components HIRA and ASF1a have been implicated in regulating senescence ${ }^{12,39}$, our studies suggest for the first time that their substrate $\mathrm{H} 3.3$, as well as its nucleosomeincorporated cleaved product $\mathrm{H} 3.3 \mathrm{cs} 1$, play an important role in 
promoting senescence. Consistent with data from others ${ }^{25}$, our immunofluorescence data suggest that H3.3 is largely excluded from SAHF. Interestingly, we also show that CTSL1 inhibition/ knockdown leads to defective SAHF formation during OIS, raising the possibility that $\mathrm{H} 3$ tail cleavage may play a role in the reorganization of chromatin during senescence.

Here, we provide evidence that $\mathrm{H} 3.3 \mathrm{cs} 1$ can mediate gene expression pathways consistent with senescence. Specifically, we found that $\mathrm{H} 3.3 \mathrm{cs} 1$ mediates the transcriptional repression of a large subset of genes previously shown to be downregulated by RB and JARID1A/1B during senescence ${ }^{15}$. Similar to RB- and JARID1-regulated genes, a large subset of $\mathrm{H} 3.3 \mathrm{cs} 1$-downregulated genes lose $\mathrm{H} 3 \mathrm{~K} 4 \mathrm{me} 3$ and are primarily E2F targets involved in cell cycle progression. We find that such transcriptional suppression occurs even at very early time points after introduction of $\mathrm{H} 3.3 \mathrm{cs} 1$ (for example, 3 days; Fig. 5a,b), and is coupled to a lack of H3K4me3 promoter occupancy (Fig. 5e), suggesting that these genes may be direct targets of the cleaved histone.

Finally, we propose that HUCA incorporates H3.3 during senescence in a replication-independent manner, likely replacing $\mathrm{H} 3.1$ at genomic locations including the promoter or regulatory regions of cell cycle-promoting genes (Fig. 6). JARID1A/1Bmediated demethylation and CTSL1-mediated H3.3 tail cleavage may cooperate or work independently in the removal of $\mathrm{H} 3 \mathrm{~K} 4 \mathrm{me} 3$, thus contributing to the transcriptional suppression of these genes (Fig. 6). Owing to the significant overlap between JARID1A/1B and H3.3cs1 target genes, histone demethylation and cleavage mechanisms may act synergistically at neighbouring nucleosomes or at the very same nucleosomes. Furthermore, because $\mathrm{H} 3$ tail cleavage is a more stable chromatin modification than enzymatic demethylation, the removal of $\mathrm{H} 3 \mathrm{~K} 4 \mathrm{me} 3$ via histone proteolysis may contribute to long-term repression of proliferation-promoting genes and thus, the irreversible nature of senescence. Collectively, our studies have identified a novel biological function for the proteolytic cleavage of $\mathrm{H} 3.3$ in senescence, and we look forward to studies that characterize the cleavage of $\mathrm{H} 3$ and other histones/histone variants in their respective biological contexts.

\footnotetext{
Methods

Cell culture. IMR90 fibroblasts were grown in Dulbecco's modified Eagle's medium supplemented with $10 \%$ foetal bovine serum and $1 \%$ penicillin/streptomycin. Normal human melanocytes were isolated from the epidermis of neonatal foreskins and cultured in Melanocyte Growth Media 254 supplemented with Human Melanocyte Growth Supplement (Life Technologies).
}

Plasmids and shRNA. The following retroviral and lentiviral constructs were used in this study: pLNCX2-neo (ER::RAS $\left.{ }^{12}\right)^{19}$, HIV-CS-CGBRAFE600-puro and pLL3.7-GFP (Addgene). Human full-length and cleaved histone $\mathrm{H} 3$ constructs were cloned into the retroviral vector $\mathrm{pBabe}$-puro (Addgene) with a C-terminal Flag tag and expressed in IMR90 cells. Point mutations of $\mathrm{H} 3.3 \mathrm{cs} 1$ were synthesized by Invitrogen (GeneArt) and subsequently cloned into the pBabe vector (BamHIEcoRI). For shRNA studies, IMR90 cells were infected with lentiviral constructs encoding CTSL1 shRNAs (Open Biosystems, RHS4533-NM_001912), ASF1a shRNA (The RNAi Consortium validated TRCN0000074269), shDaxx (TRCN0000003800), shUBN1 (TRCN0000017813) or luciferase shRNA by standard procedures. Infected cells were selected using either $1 \mu \mathrm{g} \mathrm{ml}^{-1}$ puromycin or $800 \mu \mathrm{g} \mathrm{ml}^{-1}$ neomycin.

OIS. OIS was performed as described ${ }^{19}$. Briefly, IMR90 human diploid fibroblasts were transduced with a tamoxifen-inducible H-RAS ${ }^{\mathrm{V} 12}$ construct (pLNCX2-neo ER::RAS ${ }^{\mathrm{V} 12}$ ). A final concentration of $100 \mathrm{nM} 4 \mathrm{OHT}$ was used to induce senescence over a 2 -week period. Senescence induction with BRAF ${ }^{\mathrm{V} 600 \mathrm{E}}$ in melanocytes was performed as described ${ }^{6}$. Briefly, human neonatal primary melanocytes (passage 2) were transduced with the lentiviral constructs HIV-CSCGBRAFE600-puro or pLL3.7-GFP control. BRAFV600E-expressing cells were selected with puromycin at $1 \mu \mathrm{g} \mathrm{ml}^{-1}$ for 3-4 days and grown in supplemented Melanocyte Growth Media 254 for a total of 21 days post infection.
CTSL1 inhibition. For CTSL1 inhibition studies, IMR90 ER::RAS V12 cells were cultured under growing conditions or induced to senesce with $100 \mathrm{nM} 4 \mathrm{OHT}$ in the presence of either DMSO or 1-5 $\mu \mathrm{M}$ CTSL1 inhibitor (Calbiochem, 219421-1MG).

Chromatin fractionation and histone acid extraction. Chromatin fractionation was performed as described ${ }^{40}$. Briefly, cell pellets were resuspended in buffer A (10 mM Hepes $\mathrm{pH}=7.9,10 \mathrm{mM} \mathrm{KCl}, 1.5 \mathrm{mM} \mathrm{MgCl}_{2}, 0.34 \mathrm{M}$ sucrose, $10 \%$ glycerol, $0.1 \%$ triton-X, supplemented with protease inhibitor cocktail (Calbiochem, 539131) and $1 \mathrm{mM}$ dithiothreitol (DTT)) and incubated on ice for 7-8 min. Nuclei were harvested by centrifugation at 4,000 r.p.m. for $5 \mathrm{~min}$, and the supernatant containing the cytoplasmic fraction was collected. Pelleted nuclei were washed once with triton-free Buffer A before incubation in no-salt buffer ( $3 \mathrm{mM}$ EDTA, $0.2 \mathrm{mM}$ EGTA, $1 \mathrm{mM}$ DTT and protease inhibitor) on ice for $40 \mathrm{~min}$. The chromatin fraction was recovered by centrifugation at 4,000 r.p.m. for $5 \mathrm{~min}$. Supernatant from this last step containing the nuclear-soluble fraction was mixed with the cytoplasmic fraction to obtain the chromatin-free fraction. Histone acid extraction was performed as described ${ }^{41}$. Briefly, fresh frozen nevi were ground in hypotonic lysis buffer (10 mM Tris- $\mathrm{HCl} \mathrm{pH} 8.0,1 \mathrm{mM} \mathrm{KCl}, 1.5 \mathrm{mM} \mathrm{MgCl}_{2}, 0.5 \% \mathrm{NP}-40$, $1 \mathrm{mM}$ DTT, protease inhibitors), lysed for $30 \mathrm{~min}$ on ice and spun at 1,500 r.p.m. for $10 \mathrm{~min}$. Nuclei were resuspended in $0.4 \mathrm{~N} \mathrm{H}_{2} \mathrm{SO}_{4}$ and rocked overnight at $4{ }^{\circ} \mathrm{C}$. Histones were subsequently precipitated in $20 \%$ TCA (final concentration), spun at maximum speed for $10 \mathrm{~min}$ and washed sequentially with acidified-acetone and acetone.

Mnase digestion and immunoblots. Micrococcal nuclease treatment was performed as described ${ }^{42}$. Immunoblots were performed with the following antibodies: H3 C terminus (Millipore, 05-928 1:1,000), H3K4me3 (Millipore, 05-745 1:1,000), H3K9me3 (Millipore, 07-442 1:1,000), H3K14ac (Millipore, 07-353 1:5,000), H3K18ac (Millipore, 07-354 1:5,000), H3K27me3 (Millipore, 07-449 1:1,000), H3K36me2 (Millipore, 07-369 1:1,000), H3.3 (Millipore, 09-838 1:500), H4K20me3 (Millipore, 07-463 1:1,000), H3cs1 (kind gift of Dr David C. Allis 1:1,000), macroH2A1 (Millipore, 07-219 1:1,000), macroH2A2 (Bernstein Lab 1:1,000), H2A.Z (Millipore, 07-594 1:1,000), H2A.X S139ph (Cell Signaling, 97185 1:1,000), $\beta$-Actin (Sigma, A5441 1:5,000), CTSL1 (R\&D systems, AF952 1:1,000), Flag (Sigma, F7425 1:1,000), MEK2 (BD BioScience, 610235 1:1,000), H-RAS (Santa Cruz Biotechnology, sc-29 1:1,000), p16 (Santa Cruz Biotechnology, sc-468 1:500), p21 (BD BioScience, 556431 1:1,000), p53 (Santa Cruz Biotechnology sc-126, 1:1,000). See Supplementary Fig. 13 for original scans of key immunoblots.

Senescence assays and gene expression. All senescence assays including detection of SA- $\beta$-gal, EdU incorporation, SAHF and gene expression changes were performed as described ${ }^{14}$. For colony formation assays, 10,000 cells per condition were plated in six-well plates and grown for 10 days post selection. Gene expression changes were measured using quantitative PCR with reverse transcription analysis. Briefly, total RNA was extracted using the RNeasy kit (Qiagen) followed by reverse transcription using SuperScript II (Life Technologies). PCRs were performed on a 7500 Fast Real Time system (Applied Biosystems) using SYBR Green Master Mix (Roche Applied Science). All values were normalized to glyceraldehyde 3-phosphate dehydrogenase and relative to the control using the $\Delta$ CT method. All quantitative PCRs were performed in triplicate. Primer sequences can be found in Supplementary Table 1.

Nevi collection. Approval to collect benign nevi was granted by the Division of Dermatopathology, Icahn School of Medicine at Mount Sinai (project number 080964).

Immunofluorescence. Immunofluorescence staining was performed as described previously ${ }^{40}$. Briefly, cells were placed on sterilized covered slips, fixed in $4 \%$ paraformaldehyde, permeabilized with phosphate-buffered saline $+0.1 \%$ or $0.5 \%$ Triton X-100 (PBST), blocked in PBST $+1 \%$ bovine serum albumin or $0.5 \%$ normal goat serum and stained in the same buffer with primary and secondary antibodies before mounting onto slides. Cells were analysed using the Zeiss, Axio Imager.Z1 or Leica DMI6000B SP5 confocal system with LAS AF software. Flag antibody (Cell Signaling 2368, 1:700) and H3K9me3 (CMA318, 1:100, kind gift of Hiroshi Kimura) ${ }^{43}$ were used for staining confocal images of SAHF. DAPI was used to visualize SAHF.

RNA-seq. Total RNA samples were isolated using the RNeasy kit (Qiagen) and prepared at the Icahn School of Medicine at Mount Sinai Genomics Core Facility for poly A library construction and sequencing on IlluminaHiSeq 2000. All raw RNA-sequencing reads were mapped to the human genome (hg19) with TopHat v2.0.3 (ref. 44) coupled with Bowtie2 (ref. 45) with default parameters. Transcriptomes were assembled and fragments per kilo-base per million reads for each gene were computed with Cufflinks v2.1.1 (ref. 46). Differentially expressed genes were identified using $\log 2$ fold change of fragments per kilo-base per million 
read values for all samples in pair-wise combinations. The cutoff 1.6 was used to determine lists of differentially expressed genes, as it produces lists in the hundreds range, which is suitable for enrichment and overlap analyses. ChIP enrichment analysis was carried out using the ChEA gene-set library ${ }^{30}$ implemented within Enrichr ${ }^{47}$. The Fisher exact test with the Benjamini-Hochberg correction for multiple hypotheses testing was used to perform enrichment analyses. GOenrichment analysis was carried out using DAVID ${ }^{48}$ with default parameters. Hierarchical clustering was performed using the $\log 2$ fold change of the differentially expressed genes in senescence or the H3.3cs1 samples versus the growing control sample using MATLAB (Natick, MA). Venn diagrams were plotted to demonstrate the overlap between the identified differentially expressed genes in this study and JARID1A/1B and RB targets, as well as H3K4me3-depleted genes during senescence extracted from Chicas et al. ${ }^{15}$ The $-\log (P)$ threshold for H3K4me3-depleted genes was set to 4.253 to have comparable size to the JARID1A/1B target set.

Data deposition. All RNA-Seq data sets have been deposited to NCBI's Gene Expression Omnibus with the deposition number GSE55949.

Native chromatin immunoprecipitation. Chromatin from IMR90 cells expressing H3.1FL and H3.3cs1 was digested with MNase and used for native ChIP using H3K4me3 antibody (Abcam, ab1012), and immunoprecipitated DNA was used for qPCR essentially as described ${ }^{42}$. Primers were designed $<1 \mathrm{~kb}$ downstream of the transcription start site (TSS) and controls were designed within intergenic regions. Primer sequences can be found in Supplementary Table 2.

\section{References}

1. Collado, M. et al. Tumour biology: senescence in premalignant tumours. Nature 436, 642 (2005)

2. Collado, M. \& Serrano, M. Senescence in tumours: evidence from mice and humans. Nat. Rev. Cancer 10, 51-57 (2010).

3. Hayflick, L. \& Moorhead, P. S. The serial cultivation of human diploid cell strains. Exp. Cell Res. 25, 585-621 (1961).

4. Serrano, M., Lin, A. W., McCurrach, M. E., Beach, D. \& Lowe, S. W. Oncogenic ras provokes premature cell senescence associated with accumulation of p53 and p16INK4a. Cell 88, 593-602 (1997).

5. Hemann, M. T. \& Narita, M. Oncogenes and senescence: breaking down in the fast lane. Genes Dev. 21, 1-5 (2007).

6. Michaloglou, C. et al. BRAFE600-associated senescence-like cell cycle arrest of human naevi. Nature 436, 720-724 (2005).

7. Salama, R., Sadaie, M., Hoare, M. \& Narita, M. Cellular senescence and its effector programs. Genes Dev. 28, 99-114 (2014).

8. Voorhoeve, P. M. \& Agami, R. The tumor-suppressive functions of the human INK4A locus. Cancer Cell 4, 311-319 (2003).

9. Chicas, A. et al. Dissecting the unique role of the retinoblastoma tumor suppressor during cellular senescence. Cancer Cell 17, 376-387 (2010).

10. Rai, T. S. \& Adams, P. D. Lessons from senescence: chromatin maintenance in non-proliferating cells. Biochim. Biophys. Acta 1819, 322-331 (2011)

11. Narita, M. et al. $\mathrm{Rb}$-mediated heterochromatin formation and silencing of $\mathrm{E} 2 \mathrm{~F}$ target genes during cellular senescence. Cell 113, 703-716 (2003).

12. Zhang, R. et al. Formation of MacroH2A-containing senescence-associated heterochromatin foci and senescence driven by ASFla and HIRA. Dev. Cell 8, 19-30 (2005).

13. Narita, M. et al. A novel role for high-mobility group a proteins in cellular senescence and heterochromatin formation. Cell 126, 503-514 (2006).

14. Chandra, T. et al. Independence of repressive histone marks and chromatin compaction during senescent heterochromatic layer formation. Mol. Cell 47, 203-214 (2012)

15. Chicas, A. et al. H3K4 demethylation by Jaridla and Jarid1b contributes to retinoblastoma-mediated gene silencing during cellular senescence. Proc. Natl Acad. Sci. USA 109, 8971-8976 (2012).

16. Bannister, A. J. \& Kouzarides, T. Histone methylation: recognizing the methyl mark. Methods Enzymol. 376, 269-288 (2004).

17. Allis, C. D., Bowen, J. K., Abraham, G. N., Glover, C. V. \& Gorovsky, M. A. Proteolytic processing of histone $\mathrm{H} 3$ in chromatin: a physiologically regulated event in Tetrahymena micronuclei. Cell 20, 55-64 (1980).

18. Duncan, E. M. et al. Cathepsin L proteolytically processes histone $\mathrm{H} 3$ during mouse embryonic stem cell differentiation. Cell 135, 284-294 (2008).

19. Young, A. R. et al. Autophagy mediates the mitotic senescence transition. Genes Dev. 23, 798-803 (2009).

20. Hiwasa, T. \& Sakiyama, S. Nuclear localization of procathepsin L/MEP in rastransformed mouse fibroblasts. Cancer Lett. 99, 87-91 (1996).

21. Goulet, B. et al. A cathepsin L isoform that is devoid of a signal peptide localizes to the nucleus in $\mathrm{S}$ phase and processes the $\mathrm{CDP} / \mathrm{Cux}$ transcription factor. Mol. Cell 14, 207-219 (2004).

22. Ishidoh, K. et al. Multiple processing of procathepsin L to cathepsin L in vivo. Biochem. Biophys. Res. Commun. 252, 202-207 (1998).
23. Galvani, A. et al. In vivo study of the nucleosome assembly functions of ASF1 histone chaperones in human cells. Mol. Cell. Biol. 28, 3672-3685 (2008).

24. Adams-Cioaba, M. A., Krupa, J. C., Xu, C., Mort, J. S. \& Min, J. Structural basis for the recognition and cleavage of histone $\mathrm{H} 3$ by cathepsin L. Nat. Commun. 2, 197 (2011).

25. Corpet, A., Olbrich, T., Gwerder, M., Fink, D. \& Stucki, M. Dynamics of histone H3.3 deposition in proliferating and senescent cells reveals a DAXX-dependent targeting to PML-NBs important for pericentromeric heterochromatin organization. Cell Cycle 13, 249-267 (2014).

26. Ahmad, K. \& Henikoff, S. The histone variant H3.3 marks active chromatin by replication-independent nucleosome assembly. Mol. Cell 9, 1191-1200 (2002).

27. Goldberg, A. D. et al. Distinct factors control histone variant H3.3 localization at specific genomic regions. Cell 140, 678-691 (2010).

28. Drané, P., Ouararhni, K., Depaux, A., Shuaib, M. \& Hamiche, A. The deathassociated protein DAXX is a novel histone chaperone involved in the replication-independent deposition of H3.3. Genes Dev. 24, 1253-1265 (2010)

29. Lewis, P. W., Elsaesser, S. J., Noh, K.-M., Stadler, S. C. \& Allis, C. D. Daxx is an H3.3-specific histone chaperone and cooperates with ATRX in replicationindependent chromatin assembly at telomeres. Proc. Natl Acad. Sci. USA 107, 14075-14080 (2010).

30. Lachmann, A. et al. ChEA: transcription factor regulation inferred from integrating genome-wide ChIP-X experiments. Bioinformatics 26, 2438-2444 (2010).

31. Kuilman, T. \& Peeper, D. S. Senescence-messaging secretome: SMS-ing cellular stress. Nat. Rev. Cancer 9, 81-94 (2009).

32. Coppe, J. P., Desprez, P. Y., Krtolica, A. \& Campisi, J. The senescenceassociated secretory phenotype: the dark side of tumor suppression. Annu. Rev. Pathol. 5, 99-118 (2010).

33. Rodier, F. et al. Persistent DNA damage signalling triggers senescenceassociated inflammatory cytokine secretion. Nat. Cell Biol. 11, 973-979 (2009).

34. Di Micco, R. et al. Oncogene-induced senescence is a DNA damage response triggered by DNA hyper-replication. Nature 444, 638-642 (2006).

35. Bartkova, J. et al. Oncogene-induced senescence is part of the tumorigenesis barrier imposed by DNA damage checkpoints. Nature 444, 633-637 (2006).

36. Ivanov, A. et al. Lysosome-mediated processing of chromatin in senescence. J. Cell Biol. 202, 129-143 (2013).

37. Goulet, B. et al. Increased expression and activity of nuclear cathepsin L in cancer cells suggests a novel mechanism of cell transformation. Mol. Cancer Res. 5, 899-907 (2007).

38. Lacoste, N. et al. Mislocalization of the centromeric histone variant CenH3/ CENP-A in human cells depends on the chaperone DAXX. Mol. Cell 53, 631-644 (2014)

39. Zhang, R., Chen, W. \& Adams, P. D. Molecular dissection of formation of senescence-associated heterochromatin foci. Mol. Cell. Biol. 27, 2343-2358 (2007).

40. Bernstein, E. et al. Mouse polycomb proteins bind differentially to methylated histone $\mathrm{H} 3$ and RNA and are enriched in facultative heterochromatin. Mol. Cell. Biol. 26, 2560-2569 (2006).

41. Kapoor, A. et al. The histone variant macroH2A suppresses melanoma progression through regulation of CDK8. Nature 468, 1105-1109 (2010).

42. Hasson, D. et al. The octamer is the major form of CENP-A nucleosomes at human centromeres. Nat. Struct. Mol. Biol. 20, 687-695 (2013).

43. Hayashi-Takanaka, Y. et al. Tracking epigenetic histone modifications in single cells using Fab-based live endogenous modification labeling. Nucleic Acids Res. 39, 6475-6488 (2011).

44. Trapnell, C., Pachter, L. \& Salzberg, S. L. TopHat: discovering splice junctions with RNA-Seq. Bioinformatics 25, 1105-1111 (2009).

45. Langmead, B. \& Salzberg, S. L. Fast gapped-read alignment with Bowtie 2. Nat Methods 9, 357-359 (2012).

46. Trapnell, C. et al. Transcript assembly and quantification by RNA-Seq reveals unannotated transcripts and isoform switching during cell differentiation. Nat. Biotechnol. 28, 511-515 (2010).

47. Chen, E. Y. et al. Enrichr: interactive and collaborative HTML5 gene list enrichment analysis tool. BMC Bioinformatics 14, 128 (2013).

48. Huang da, W., Sherman, B. T. \& Lempicki, R. A. Systematic and integrative analysis of large gene lists using DAVID bioinformatics resources. Nat. Protoc 4, 44-57 (2009).

\section{Acknowledgements}

We thank Elizabeth Duncan for critical reading of this manuscript. We thank Kajan Ratnakumar, Zulekha Qadeer, Hayley Zullow, Thomas Strub and Alexandre GasparMaia for technical assistance. David Allis, Scott Lowe, Daniel Peeper, Jesus Gil, Hiroshi Kimura and Sandra Hake kindly provided reagents for these studies. We thank CRUK CI microscope facility for technical support. We also thank Madelaine Haddican, Shelbi Jim On and Giselle Singer in the Department of Dermatology for assistance with benign nevi collection, and Dr Mark Lebwohl for his continued support. This work was supported by 
Award Number T32CA078207 from the National Cancer Institute to L.F.D., University of Cambridge, Cancer Research UK, Hutchison Whampoa and the Human Frontier Science Programme to M.N., R01GM098316 and P50GM071558 to A.M. and by funds from The Ellison Medical Foundation New Scholar Award, Developmental Research Pilot Project Programme (ISMMS) and NCI/NIH R01CA154683 to E.B.

\section{Author contributions}

L.F.D. and E.B. conceived this study. L.F.D. and A.K. identified H3 cleavage. L.F.D. and A.R.J.Y. performed senescence experiments with associated assays, CTSL studies and immunofluorescence. L.F.D. performed immunoblots and RT-qPCR experiments with assistance from T.P., N.R.M. and A.R.J.Y. T.P. performed melanocyte isolation and nevi histone extractions. Z.W. and H.-A.W. cloned H3 constructs and $\mathrm{H} 3.3 \mathrm{csl}$ constructs. H.-A.W. assisted with figures and illustrations. Z.W., Y.K. and A.M. performed RNA-seq and statistical analysis. L.F.D. and D.H. performed native ChIP. L.F.D., A.R.J.Y., D.H., M.N. and E.B. designed experiments and interpreted results. E.B. and L.F.D. wrote the manuscript with contributions from all other coauthors.

\section{Additional information}

Supplementary Information accompanies this paper at http://www.nature.com/ naturecommunications

Competing financial interests: The authors declare no competing financial interests.

Reprints and permission information is available online at http://npg.nature.com/ reprintsandpermissions/

How to cite this article: Duarte, L. F. et al. Histone H3.3 and its proteolytically processed form drive a cellular senescence programme. Nat. Commun. 5:5210 doi: 10.1038/ncomms6210 (2014) 